\title{
Reabilitação da capsulite adesiva após tratamento conservador de fratura proximal de úmero: Relato de caso
}

\section{Rehabilitation of adhesive capsulitis after conservative treatment of proximal humerus fracture: Case report}

DOI: $10.46919 / \operatorname{archv} 1 \mathrm{n} 6-001$

Recebimento dos originais: 01/09/2020

Aceitação para publicação: 30/10/2020

\section{Vanessa da Silva Caiado}

Fisioterapeuta Especialista em Fisioterapia traumato -ortopédica / Universidade Gama Filho Instituição atual: Universidade do estado do Rio de Janeiro (UERJ) Policlínica Piquet Carneiro Avenida Marechal Rondon, 381- São Francisco Xavier. Cep: 20950003. Rio de Janeiro - RJ, Brasil E-mail: vancaiado83@gmail.com

\section{Cláudia Zornoff Gavazza}

Mestre em Saúde coletiva / Área da saúde da criança e da mulher/ Instituto Fernandes Figueira Instituição atual: Universidade do estado do Rio de Janeiro (UERJ) Policlínica Piquet Carneiro Avenida Marechal Rondon, 381- São Francisco Xavier. Cep: 20950003. Rio de Janeiro - RJ, Brasil

E-mail: claudiazgavazza@gmail.com

\section{Guilherme Salcedo Areunete}

Fisioterapeuta Especialista em Fisioterapia esportiva /Conselho federal de fisioterapia e terapia ocupacional(COFFITO)

Instituição atual: Universidade do estado do Rio de Janeiro (UERJ)/ Policlínica Piquet Carneiro Avenida Marechal Rondon, 381- São Francisco Xavier. Cep: 20950003. Rio de Janeiro - RJ, Brasil

E-mail: guilhermeareunete@gmail.com

\section{Mariana Alonso Monteiro Bezerra}

Mestre em Saúde Coletiva / Área de concentração Epidemiologia / Instituto de Medicina Social / Universidade do Estado do Rio de Janeiro.

Instituição atual: Universidade do estado do Rio de Janeiro (UERJ)/ Hospital Universitário Pedro Ernesto Boulevard vinte e oito de Setembro, 77- Vila Isabel. Rio de Janeiro -RJ, Brasil

E-mail: ft.marianaalonso@gmail.com

\section{Letícia Del castillo Mathias}

Mestre em ciências / Departamento de pós graduação em ciências médicas / UERJ Instituição atual: Universidade do estado do Rio de Janeiro (UERJ) / Hospital Universitário Pedro Ernesto.

Boulevard vinte e oito de Setembro, 77- Vila Isabel. Rio de Janeiro -RJ, Brasil

E-mail: leticia.castillo.mathias@gmail.com 


\title{
Adalgisa Ieda Maiworm Bromershenkel
}

Doutora em ciências médicas /Universidade do Estado do Rio de Janeiro

Instituição atual: Universidade do estado do Rio de Janeiro (UERJ) / Policlínica Piquet Carneiro -

Avenida Marechal Rondon, 381- São Francisco Xavier. Cep: 20950003. Rio de Janeiro - RJ, Brasil

E-mail: aibmaiworm@gmail.com

\section{RESUMO}

Introdução: A capsulite adesiva é uma condição de etiologia incerta e pode estar associada a mecanismos pós traumáticos seguidos de longos períodos de imobilização causando dor e redução da amplitude de movimento (ADM) da articulação do ombro.

Objetivo: Descrever as técnicas e os resultados de um protocolo fisioterapêutico utilizado em um caso de capsulite adesiva após tratamento conservador de fratura proximal de úmero esquerdo.

Método: $\mathrm{O}$ indivíduo foi submetido a 2 sessões de fisioterapia por semana totalizando 25 sessões. Foram realizadas técnicas visando o aumento da amplitude de movimento como alongamento de cápsula articular, facilitação neuro muscular proprioceptiva e exercícios ativos com e sem carga.

Resultados e conclusão: Após o tratamento realizado houve aumento significativo da amplitude de movimento da articulação do ombro e redução da dor. O protocolo fisioterapêutico utilizado para reabilitação de capsulite adesiva após tratamento conservador de fratura proximal de úmero no caso relatado foi eficaz.

Palavras-chaves: capsulite adesiva, reabilitação, fratura proximal de úmero.

\begin{abstract}
Background: Adhesive capsulitis is a condition of uncertain etiology and may be associated with posttraumatic mechanisms followed by long periods of immobilization causing pain and reduced range of motion of the shoulder joint.

Objective: To describe and report the techniques and results of a physiotherapy protocol used in one case of adhesive capsulitis after conservative treatment of a proximal fracture of the left humerus.

Method: The individual was submitted to 2 physiotherapy sessions per week, totaling 25 sessions. The techniques were performed to increase the range of motion such as stretching of the joint capsule, proprioceptive neuro muscular facilitation besides weight and non weight bearing exercises.

Results and conclusion: After the treatment, there was a significant increase in the range of motion of the shoulder joint and a reduction in pain. The physical therapy protocol used for rehabilitation of adhesive capsulitis after conservative treatment of proximal humerus fracture in the case reported was effective.
\end{abstract}

Keywords: adhesive capsulitis, rehabilitation, proximal humerus fracture.

\section{INTRODUÇÃO}

A Capsulite Adesiva (CA) é caracterizada por dor e limitação de amplitude de movimento (ADM) passivo e ativo da articulação do ombro, sendo mais comum no gênero feminino na faixa etária de 45 a 65 anos (1). A fratura de úmero é relatada como causa secundária extrínseca para a CA. A fisioterapia tem como objetivos: diminuir a dor, aumentar a ADM e a força muscular.

Objetivo/Relato de caso

Paciente, 73 anos, gênero masculino, com histórico de fratura impactada de úmero proximal esquerdo. Realizou tratamento conservador com imobilização por tipóia por cinco semanas. À avaliação 
do ombro apresentou: $\mathrm{ADM}$ - flexão: $70^{\circ}$; rotação externa: 70॰; rotação interna: $80^{\circ}$; extensão: $60 \circ$; e abdução 80॰. Força muscular: grau 4 em adutores, abdutores, rotadores internos e externos, flexores e extensores. Escala Visual Analógica de dor: 5 ao final da flexão.

\section{MÉTODO / DISCUSSÃO}

A reabilitação ocorreu em um período total de 25 sessões que foram realizadas duas vezes por semana. $\mathrm{O}$ paciente foi submetido às técnica de alongamento da cápsula anterior, posterior e inferior para aumento de rotação externa, flexão e rotação interna de ombro respectivamente. Exercício ativo livre para flexão de ombro em decúbito lateral direito. Facilitação neuromuscular proprioceptiva em cadeia cinética fechada na posição sentada com o objetivo de estabilidade e controle motor da cintura escapular. Mobilização passiva articular da articulação glenoumeral com deslizamento inferior da cabeça do úmero. Exercício ativo livre para flexão de ombro através de polia e com bastão. Exercício ativo resistido de adutores, abdutores, flexores, extensores e rotadores internos e externos de ombro visando o fortalecimento muscular.

\section{RESULTADO/CONCLUSÃO}

Após 25 sessões o paciente apresentou os seguintes resultados: flexão de ombro 130॰, extensão 60॰ ,abdução $110^{\circ}$,rotação interna $90^{\circ}$,rotação externa 90॰.Força muscular grau 5 nos músculos avaliados e redução da dor na escala visual analógica de 5 para 0 durante a flexão de ombro. A fisioterapia mostrou melhora para redução da dor, aumento da mobilidade articular e força muscular em paciente com capsulite adesiva após período de imobilização de fratura impactada proximal de úmero em tratamento conservador.

\section{REFERÊNCIAS}

1-Kelly MJ,Shaffer MA.Shoulder pain and mobility deficits Adhesive Capsulitis.Clinical Practice Guidelines linked to the International Classification of functioning,disability,and health from the orthopaedic Section of the American physical therapy association.J Orthop Sports Phys Ther.2013;43(5):A1-A31.

2-Hane J,Brophy RH,Barker JU. Manegement of proximal humeral fractures based on current literature.J Bone Joint Surg .2007;89:44-58.

3- Page MJ, Green S, Kramer S., Johnston RV, McBain B. et al., Manual therapy and exercise for adhesive capsulitis (frozen shoulder) (Review)., Cochrane Library, 2014. 